\title{
The Effects of Massage on Pain, Stiffness, and Fatigue Levels Associated with Ankylosing Spondylitis: A Case Study
}

\author{
Rosemary Chunco, LMT, BA, MSc \\ Shamrock Therapeutics LLC, Plano, Texas, USA
}

Objectives: To study the effects of massage on pain, stiffness, and fatigue in a patient recently diagnosed with ankylosing spondylitis (AS).

Methods: A 47-year-old woman with AS diagnosed 11 months earlier received 7 massages across a 28 -day period. Her pain, stiffness, and fatigue were recorded using visual analogue scales daily during the study period. Spinal mobility was measured at each massage session with finger-to-floor measurements for both forward and lateral flexion. The client also used a daily journal to supply pertinent information on quality of life.

Results: Improvement was recorded in all dependent variables, with stiffness intensity showing the greatest improvement, to a final value of 0.75 from an initial value of 5 . Duration of stiffness also showed improvement, to a final value of 1.2 from an initial value of 3.5. Improvement was also found in general pain (final value: 1 ; initial value: 4 ), fatigue (final value: 1.5; initial value 5), and forward and lateral flexion (forward flexion distance-final: 4 inches; initial: 6 inches; lateral flexion, left distance: final, 16.5 inches; initial, 21 inches; right distance: final, 16.5 inches; initial, 20.5 inches).

Conclusions: Massage shows promise as a treatment for symptoms associated with AS. Further study is needed to validate these effects and to determine the feasibility of massage as an adjunct to standard care for AS patients with mild-tomoderate symptoms of AS.

KEYWORDS: Ankylosing spondylitis, massage therapy, fatigue, pain

\section{INTRODUCTION}

Ankylosing spondylitis (AS)—sometimes called rheumatoid spondylitis or Marie-Strümpell diseaseis a chronic and systemic rheumatic disease that presents as inflammation of the vertebrae and joints. The exact cause of AS is unknown, but the hypothesis is that the body's mechanisms for coping with inflammation lead to abnormal bone formation (syndesmophytes) through ossification, which can eventually lead to spinal fusion ${ }^{(1)}$. The resultant stiffening of the joints attributable to that fusion is called ankylosis, and the extent of the fusion can vary from fusion of the pelvic bones only, to fusion of the entire spine. Enthesitis (inflammation at a site where tendon attaches to bone) may lead to painful swelling and tenderness (Figure 1). The location and prevalence of enthesitis is of major significance for quality of life in a person with AS, with effects that range from sleep disturbance to anxiety, depression, and loss of income because of absence from work $^{(2,3)}$. In some cases, AS goes into spontaneous remission, followed by periodic acute phases. A common initial site for enthesitis is the sacroiliac joint, with sacroiliitis being one of the main symptoms involved in the initial diagnosis ${ }^{(4)}$. Progression of AS can lead to significant postural changes, including loss of normal lumbar lordosis, forward positioning of the neck, and exaggerated kyphosis ${ }^{(5)}$.

The main area affected by AS is the spine, but given that AS is systemic and rheumatic, other areas may
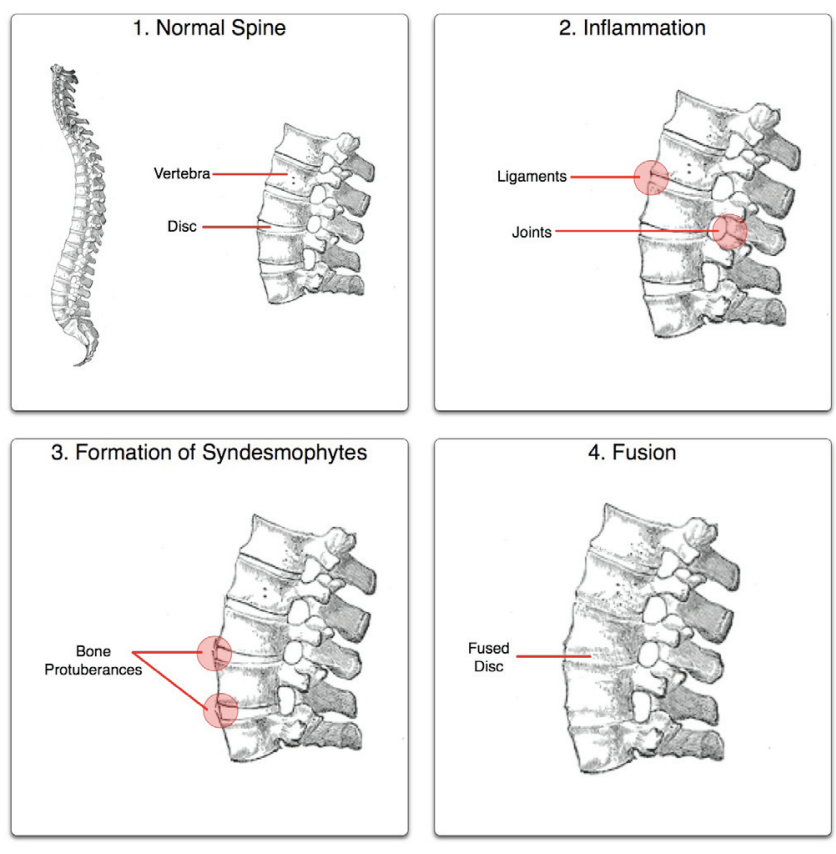

FIGURE 1. The ankylosis process (reproduced under the terms of the GNU Free Documentation License. Source: http://en.wikipedia.org/ wiki/File:Ankylosing_process.jpg). 
also develop pain or stiffness, including hips, knees, ankles, shoulders, and ribs. Hip pain can be a result of inflammation of the subchondral bone marrow. This inflammation is different from classical spine problems in that it is not formation of new bone, but an erosive process that can often lead to hip replacement ${ }^{(6)}$. Furthermore, AS can affect organs such as the eyes, heart, and kidneys; lung capacity can also be restricted because of the effects of AS on the ribcage.

It is estimated that 350,000 people in the United States have AS, and internationally, AS is thought to occur in $0.1 \%$ of the general population ${ }^{(7,8)}$. Although the cause of AS is unknown, there is evidence for a genetic link to the disease, and people with AS have a high occurrence of human leukocyte antigen B27 in tissue. Individuals with this antigen may be predisposed to developing AS, with disease onset being triggered by an environmental factor ${ }^{(9)}$. One theorized trigger for the development of AS may be connected to bacteria in the digestive tract ${ }^{(10)}$. Accurate diagnosis is often delayed because the early stages of AS mimic other conditions.

Symptoms of AS often include

- pain and stiffness in the lower back, especially the sacroiliac joint;

- pain in the gluteal muscles, which is usually worse when waking in the morning;

- persistent pain for 3 months or longer;

- stiffness, which is worse in the morning; and

- increased fatigue.

Daily stiffness, pain, and fatigue are the three most common symptoms and are reported in up to $70 \%$ of patients ${ }^{(11)}$. More men than women are affected, and AS has a tendency to affect women differently than men. A later age of onset in women has been observed, together with milder and longer asymptomatic phases between active flare-ups and a lower incidence of "bamboo spine" (the term given to the fusion and rigidity characterisitics).

Treatment for AS usually includes medication and physical therapy. Prescribed medication is usually an immunosuppressant or anti-inflammatory. Established evidence shows that exercise and physical therapy are helpful for the condition ${ }^{(12)}$, with one of the main goals being the development of strong back muscles and the maintenance of proper posture. Studies of balneotherapy (bathing in mineral springs) and hydrotherapy have shown little evidence for long-term effects ${ }^{(13)}$.

\section{Disease Measurement in Medical Practice}

There are 4 main measures of AS disease activity, collectively known as the "Bath indices," which were developed by a team of rheumatologists, physical therapists, and research specialists. These indices are

- the Bath AS Metrology Index, which measures movement in the cervical, dorsal, and lumbar spine; the hips; and the pelvic tissues.
- the Bath AS Functional Index, which uses 10 questions about coping with daily living to measure functional aspects of life. Most responses are recorded using a visual analogue scale (VAS).

- the Bath AS Disease Activity Index, which measures disease activity in relation to levels of fatigue, pain, morning stiffness, joint pain, and swelling, and localized tenderness.

- the Bath AS Patient Global Score, which is used in conjunction with other Bath indices as an evaluation of well-being in general ${ }^{(14)}$.

\section{Massage and AS}

Although the Spondylitis Association of America (SAA) recognizes massage as one of the alternative treatments that may help sufferers, little research has been published on the use of massage alone. Notably, the SAA quotes a physical therapist: "In all my years of experience as a physical therapist, I have never known massage to worsen the symptoms of inflammation in a patent with AS. Deep tissue mobilization is nearly always welcomed by those with $\mathrm{AS}$ and is usually given in combination with passive stretches and ultrasound, heat or ice"(15). However, as a cautionary note, there is also mention that massage may be a trigger for disease flare-ups. The SAA also quotes a well-known AS researcher and rheumatologist in warning against spinal manipulation with chiropractic treatments. That advice is consistent with Salvo's recommendations for massage in AS, which states that compression over the ribcage should be avoided and that anklylosed joints should not be forcibly mobilized ${ }^{(16)}$. Salvo also says that kneading, stretches, and mobilization of non-ankylosed joints are appropriate within the client's tolerance level, and she recommends using pillows for positioning and support to aid the client's comfort when receiving massage.

Werner ${ }^{(17)}$ also says that massage that may exacerbate inflammation should be avoided, but that massage can help with mobility of the spine. This author also addresses the need for caution in treating people with AS and for massage to be used only in conjunction with a health care team. Werner also notes that deep tissue massage is contraindicated, with proprioceptive neuromuscular facilitative stretching indicated. Swedish massage is also indicated if the AS client is not currently experiencing a flare-up. Trigger point therapy is locally contraindicated if the disease is in an acute stage, but is otherwise indicated. Werner also highlights the fact that little is known about how massage might affect AS.

A study by Durak and colleagues ${ }^{(18)}$ of the effects of massage on pain levels in a person with long-term AS indicated that massage helped significantly with pain levels over a 6-month period and had a significant impact on range of motion in the subject's neck. The conclusion reached was that symptoms were reduced 
$90 \%$ over the 6 -month period. That finding is a good indicator that massage might help with the pain associated with AS.

The present case study details the use of massage in a 47-year-old woman with AS who had stiffness, pain, and difficulty sleeping at night because of hip pain. This case study investigated the effects of massage on stiffness and pain over a 4 -week period, during which this woman received 7 massages. The clinical reasoning was to determine whether any benefit would be achieved over a short period of time with multiple sessions per week. The purpose was to measure the effects of massage on the 3 main AS symptoms: pain, stiffness, and fatigue. The client specifically mentioned that she would like to see a reduction in hip pain and stiffness. Because there is evidence that massage may help with chronic back pain ${ }^{(19)}$, the objective was to use massage to help with overall pain and to improve flexibility, with close monitoring in case massage exacerbated the condition.

\section{Client Profile}

The client, a 47-year-old woman, had been diagnosed with AS 11 months before the start of the study. A rheumatologist who performed a blood analysis made the diagnosis, although the client was not aware of the type of blood test used. She believes that she has had AS for years, with the first symptoms having appeared in her late $20 \mathrm{~s}$ and early $30 \mathrm{~s}$. Her brother has also been diagnosed with AS, but has more pronounced and severe symptoms.

The client has a relatively busy family life, with 2 small children under the age of 10 . She also runs two small businesses, one of which involves considerable sitting, which she feels may exacerbate her symptoms. She says that AS affects her quality of life in both the work and the home settings.

At the time of the study, the client was taking escitalopram for depression. She had previously been prescribed sulfasalazine (an immunosuppressant) for her AS, but because of health insurance issues, she had been unable to acquire the drug for 6 months before the start of the study. Sulfasalazine helped her pain and stiffness significantly when she was using it. At the time of the study, she was using over-the-counter nonsteroidal anti-inflammatories (NSAIDs - naproxen or ibuprofen) as needed. She also took loratadine as needed for seasonal allergies. She had had 3 diverticulitis attacks in the past, the most recent being 17 months before the start of the study.

This client indicated frequent fatigue, headaches, low back pain, hip pain, neck pain, and heel pain in her left foot, which may have been a result of surgery for a bone spur 8 months before the start of the study. Her hip pain radiated from above the greater trochanter to the anterior hip and leg to mid-thigh; this hip pain was bilateral. She had never received physical therapy for her condition. She had previously received massages, and she had found them to be helpful, but her last massage had been 2 years before the start of the study.

Having provided informed consent for the study, the client indicated that she preferred medium to deep pressure. Her desired outcomes were increased mobility, decreased stiffness, and the ability to achieve more restful sleep without being awakened by hip pain.

\section{METHODS}

Seven 60-minute massage sessions were administered over a 4-week period. A total of 8 sessions had been planned, but scheduling conflicts prevented the final session from being carried out.

The client was asked to complete a daily diary, which included a VAS $(100 \mathrm{~mm})$ to assess

- general pain (no pain to excruciating pain),

- fatigue (no fatigue to severely fatigued),

- morning stiffness intensity (no stiffness to intense stiffness), and

- morning stiffness duration ( $0-2$ hours or more).

Each daily entry also included a section in which the client could record notes on how she was feeling in general and whether she had to take over-the-counter NSAIDs, together with any additional information about her general health.

The massage therapist used finger-to-floor measurements for forward and lateral flexion to assess the client's spinal mobility before each massage session ${ }^{(20)}$.

The criteria and approach used were a mix of methods derived from the Bath index methods, using a subset of the scales from the Bath AS Disease Activity Index and the Bath AS Metrology Index evaluations. Because pain, fatigue, and stiffness are the 3 main complaints of AS, measurement tools specific to those complaints were chosen. This approach was also in keeping with the client's desired outcomes.

\section{Massage Protocol}

The massage protocol incorporated mostly Swedish strokes and stretching (consistent with published guidelines), with myofascial release and trigger point therapy introduced in later sessions. An original intention of the present study had been to standardize the treatment time for each anatomical area; however, that attempt was soon judged to be unfeasible, and timing therefore varied according to the client's circumstances at each session. Although the use of trigger point therapy had not initially been planned, that technique was found to be beneficial to the client in the cervical, back, and hip areas, and so it was introduced into the sessions gradually, with feedback provided by the client as to intensity level and whether pain levels in the areas worked showed improvement between sessions. Work on the tensor fasciae latae 
area seemed to help significantly with the hip pain, and when using trigger point therapy on that area, pain referral was to the anterior thigh.

Although back-strengthening home exercises may have been beneficial for the client, advice to that purpose could not be given, because to do so would be outside the massage therapist's scope of practice.

Figure 2 presents areas covered, techniques used, and approximate times allocated.

\section{RESULTS}

Reduction in morning stiffness and increased mobility were some of the most noticeable changes for the client over the 28-day period. Before the start of the study, the client found it necessary to use the arms of a chair to assist the move from a sitting to a standing position, and that need was one of her main complaints before the start of the study. By the end of the study, she could stand without using the chair arms to assist in the movement, which pleased her greatly. She said that she could feel the change mostly in her hips, and she noticed that morning stiffness had decreased considerably. The duration and intensity of stiffness decreased markedly (Figure 3). Overall, stiffness intensity showed improvement across the study, with a final VAS value of 0.75 compared with 5 initially. Stiffness duration also improved to a final VAS value of 1.2 from an initial value of 3.5.

Improvement was also found in forward and lateral flexion. These were determined using finger-to-floor measurements before each massage session. The client also noticed that her level of mobility was increasing after each session. Figure 4 shows the changes in forward and lateral flexion. Forward flexion improved to a final measurement of 4.0 inches from an initial measurement of 6.1 inches. Lateral flexion on the right improved to 16.5 inches from an initial measurement of 20.5 inches; and on the left, it improved to 16.5 inches from 21 inches. It should be noted that

Massage Techniques and Approximate Allocated Time
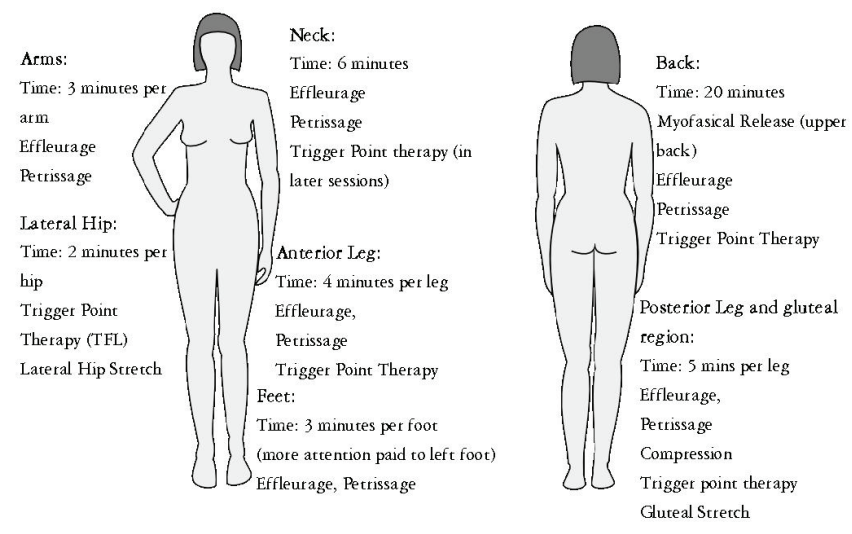

FIGURE 2. Massage techniques used during the study.

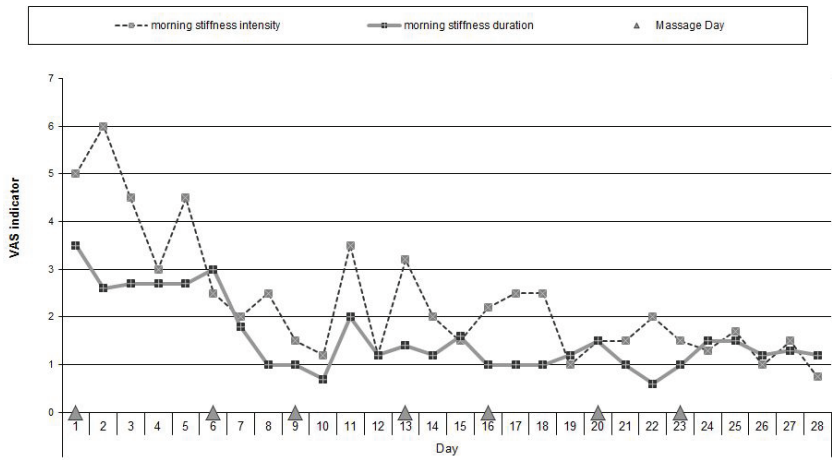

FIGURE 3. Morning stiffness intensity and duration during the study period.

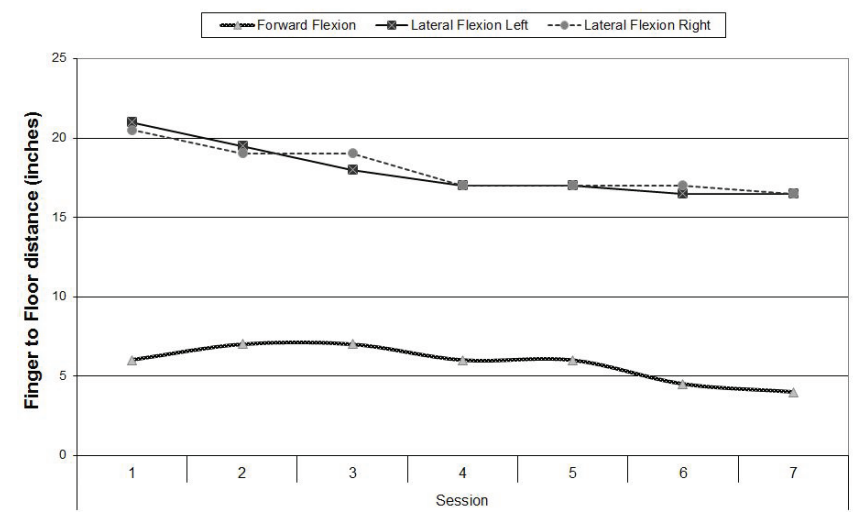

FIGURE 4. Changes in forward and lateral flexion during the study period.

forward flexion fluctuated, and measurements showed a decrease in forward flexion at the measurements taken before sessions 2 and 3 .

There was a greater noticeable fluctuation in pain and fatigue levels over the 28-day period, with the client noting that she had "bad days" periodically throughout the month. These were sometimes the result of a busy personal schedule or other circumstances that seemed to make the pain worse. For example, being seated in the car for a long period of time seemed to cause a flare-up of pain around day 11 , and a busy workday on day 20 had a similar effect. One change that was evident in the client's diary notes was an apparent considerable decrease in the hip pain, the only pain being experienced from day 21 being heel pain. The client frequently noted that, although she was marking the pain levels in the "general pain" scale, she felt pain only in her heel for 6 of the days. The massage protocol she received did not seem to have any affect on that particular pain. She said that the only relief came from wearing shoes with heels, because they took pressure off the heel. That solution could have postural implications and might lead to back pain. She also mentioned that she was sleeping better: the number of times she had awakened with pain decreased from 4 or 5 times 
per night before the study to 1 time per night at the end of the study. She also indicated that her need for NSAIDs had decreased.

Overall, the client reported being pleased with the outcomes of the massage, and she said that she plans to continue using massage to maintain levels of mobility. The massage therapist also noticed that the mood of the client seemed to improve across the study period, with the client becoming less disheartened about her condition. That observation is consistent with evidence that massage helps with depression ${ }^{(21)}$.

Figure 5 shows results for pain and fatigue levels. Pain improved to a final VAS value of 1 from an initial value of 4 . Fatigue also improved to a final VAS value of 1.5 from an initial value of 5 .

\section{DISCUSSION}

Massage had a positive outcome in this study for the 3 main areas of investigation, providing some indications that massage could be used as a complement to standard care for people with AS who have mild-to-moderate symptoms and who are not experiencing an acute flare-up. In regard to that finding, it is worth noting that, given the typical symptom presentation and the diagnostic delays associated with AS, massage therapists may well be working on clients with undiagnosed AS. The subject of the present study experienced back pain, stiffness, and fatigue for more than 15 years before being diagnosed, a fact that may inform work with clients who have similar complaints, especially female clients.

Massage helped the client in this particular study; however, it should be noted that the protocol used may not be suitable for AS patients and that it was modified to suit the client's condition. Positive outcomes may have been related to any combination of the client's more restorative sleep, the release of trigger points around the hip area, or the reduction in pain consistent with the gate control theory. A placebo effect may also come into play because the therapeutic relationship was strong as a result of execution of a study.

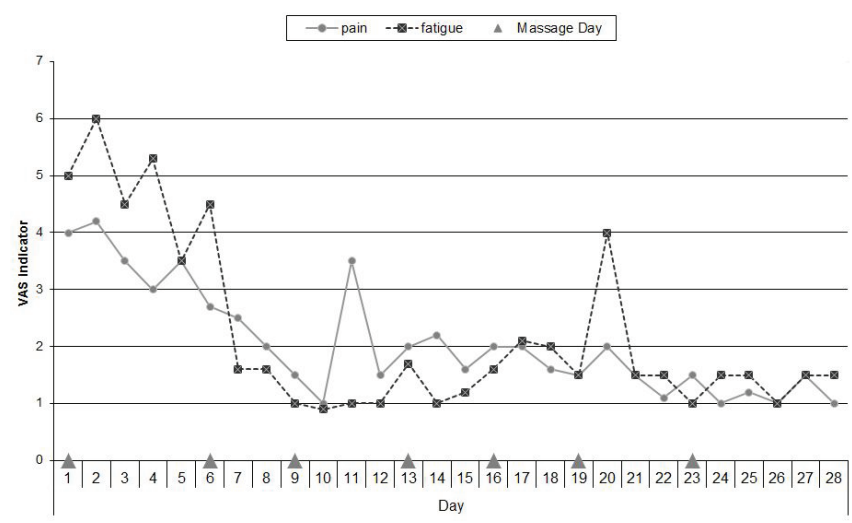

FIGURE 5. Pain and fatigue levels during the study period.
Replication of this study could provide additional useful information about the effects of massage. To gain further information, the author would advise these enhancements:

- A record of the number of hours of sleep could be added as a dependent variable to obtain a better quantitative assessment. Those data could then be used to investigate whether the level of fatigue during the day and the number of hours of sleep on the preceding night are correlated.

- Baseline and follow-up data could be added to compare averages between 3 time segments (28 days before, during, and after the intervention). Comparative periods would also show whether the benefits of massage are temporary and whether they are seen only while the client is receiving massage. Also, to provide a more meaningful quantitative assessment, it would be advisable to use one or more of the full Bath indices for each period and to compare values for each of the 3 periods.

- It would be beneficial to replicate the study in a male client, given that symptoms can present differently in men.

- In further studies, assessment by a second person of the measurements for lateral and forward flexion (both assessors being blinded to the results of the other) may ensure a higher level of accuracy. A modified finger-to-floor measurement using a stool may also be considered.

- This client had no restriction in breathing and no thoracic pain. It would be interesting and informative to conduct another case study in which massage is used over the thoracic area, with measurement of breath capacity, in a client who has ribcage restriction.

- Cross-study analysis could be conducted to provide more information if more case studies were to be done with the client. Possible patterns in outcomes could then be identified, possibly aiding in the refinement of more rigorous studies.

The author recognizes that there is still much to learn about AS, but because the disease affects quality of life for so many people, more research on how massage can help with quality of life would alone be a worthwhile endeavor.

\section{CONFLICT OF INTEREST NOTIFICATION}

The author has no conflicts of interest to declare.

\section{COPYRIGHT}

Published under the CreativeCommons AttributionNonCommercial-NoDerivs 3.0 License. 


\section{REFERENCES}

1. Baraliakos X, Listing J, Rudwaleit M, Haibel H, Brandt J, Sieper J, et al. Progression of radiographic damage in patients with ankylosing spondylitis: defining the central role of syndesmophytes. Ann Rheum Dis. 2007; 66(7):910-915.

2. Günaydin R, Göksel Karatepe A, Ceşmeli N, Kaya T. Fatigue in patients with ankylosing spondylitis: relationships with disease-specific variables, depression, and sleep disturbance. Clin Rheumatol. 2009; 28(9):1045-1051.

3. Montacer Kchir M, Mehdi Ghannouchi M, Hamdi W, Azzouz D, Kochbati S, Saadellaoui K, et al. Impact of the ankylosing spondylitis on the professional activity. Joint Bone Spine. 2009; 76(4):378-382.

4. Calin A. Ankylosing spondylitis. Medicine. 2002; 30(9):54-57.

5. Sieper J, Braun J, Rudwaleit M, Boonen A, Zink A. Ankylosing spondylitis: an overview. Ann Rheum Dis. 2002; 61(Suppl 3):iii8-iii18.

6. Vander Cruyssen B, Muñoz-Gomariz E, Font P, Mulero J, de Vlam K, Boonen A, et al. on behalf of the ASPECT-REGISPONSER-RESPONDIA Working Group. Hip involvement in ankylosing spondylitis: epidemiology and risk factors associated with hip replacement surgery. Rheumatology (Oxford). 2010; 49(1):73-81.

7. Spondylitis Association of America. Ankylosing Spondylitis Guidance Document Forwarded to the FDA. Spondylitis Association of America website. http://www.spondylitis.org/ press/as_guidance_document.aspx. Published n.d. Updated n.d. Accessed October 2010.

8. Shiel WC Jr. Ankylosing Spondylitis. MedicineNet.com website. http://www.medicinenet.com/ankylosing spondylitis/article. $\underline{\mathrm{htm}}$. Published n.d. Updated n.d. Accessed October 2010.

9. Peh WCG. Ankylosing Spondylitis. eMedicine.medscape.comwebsite. http://emedicine.medscape.com/article/386639-overview. Published n.d. Updated n.d. Accessed December 2010.

10. Benjamin R, Parham P. Guilt by association: HLA-B27 and ankylosing spondylitis. Immunol Today. 1990; 11(4):137-142.

11. O'Shea FD, Riarh R, Anton A, Inman RD. Assessing back pain: does the Oswestry Disability Questionnaire accurately measure function in ankylosing spondylitis? J Rheumatol. 2010; 37(6):1211-1213.

12. Dagfinrud H, Kvien TK, Hagen KB. The Cochrane review of physiotherapy interventions for ankylosing spondylitis. $J$ Rheumatol. 2005; 32(10):1899-1906.

13. Altan L, Bingöl U, Aslan M, Yurtkuran M. The effect of balneotherapy on patients with ankylosing spondylitis. Scand $J$ Rheumatol. 2006; 35(4):283-289.
14. Moncur C. Ankylosing spondylitis measures: the Ankylosing Spondylitis Quality of Life (ASQOL) scale, Bath Ankylosing Spondylitis Disease Activity Index (BASDAI), Bath Ankylosing Spondylitis Functional Index (BASFI), Bath Ankylosing Spondylitis Global Score (BAS-G), Bath Ankylosing Spondylitis Metrology Index (BASMI), Dougados Functional Index (DFI), Health Assessment Questionnaire for the Spondyloarthropathies (HAQ-S), and Revised Leeds Disability Questionnaire (RLDQ). Arthritis Care Res. 2003; 49(Suppl 5):S197-S209.

15. Spondylitis Association of America. Alternative Treatments. Spondylitis Association of America website. http://www. spondylitis.org/about/alternative.aspx. Published n.d. Updated n.d. Accessed October 2010.

16. Salvo SG. Mosby's Pathology for Massage Therapists. 2nd ed. Chapter 5: Musculoskeletal Pathologies, St. Louis, MO Elsevier; 2009: 103-157

17. Werner R. A Massage Therapist's Guide to Pathology. 4th ed. Chapter 3: Musculoskeletal System Conditions. Baltimore, MD: Lippincott, Williams and Wilkins; 2009: 85-218.

18. Durak E, Manion K, Martin M. The effects of segmental massage on range of motion and pain reduction in chronic inflammatory pain: a case study [Abstract]. Massage Therapy Foundation website. http://www.massagetherapyfoundation.org/ postersession.html\#The $\% 20$ Effects $\% 20$ of $\% 20$ Segmental $\% 20$ Massage $\% 20$ on $\% 20$ Range $\% 20$ of $\% 20$ Motion $\% 20$ and $\% 20$ Pain\%20Reduction\%20in\%20Chronic\%20Inflammatory\%20 Pain:\%20A\%20Case\%20Study. Published 2008. Accessed September 2010

19. Furlan AD, Imamura M, Dryden T, Irvin E. Massage for low-back pain. Cochrane Database Syst Rev. 2008; (4):CD001929.

20. National Ankylosing Spondylitis Society (NASS). The Bath Indices: Outcome Measures for Use with Ankylosing Spondylitis Patients. Mayfield, UK: NASS; 2004. [Available online at: http://www.astretch.co.uk/NASS_Bath_Indices.pdf; accessed January 2011]

21. Moyer CA, Rounds J, Hannum JW. A meta-analysis of massage therapy research. Psychol Bull. 2004; 130(1):3-18.

Corresponding author: Rosemary Chunco, 992109 West Parker Road, Plano, Texas 75023 USA. E-mail: rchunco@hotmail.com 